\title{
Primary jejunal angiosarcoma: an extremely rare tumor diagnosed by means of anterograde spiral enteroscopy
}

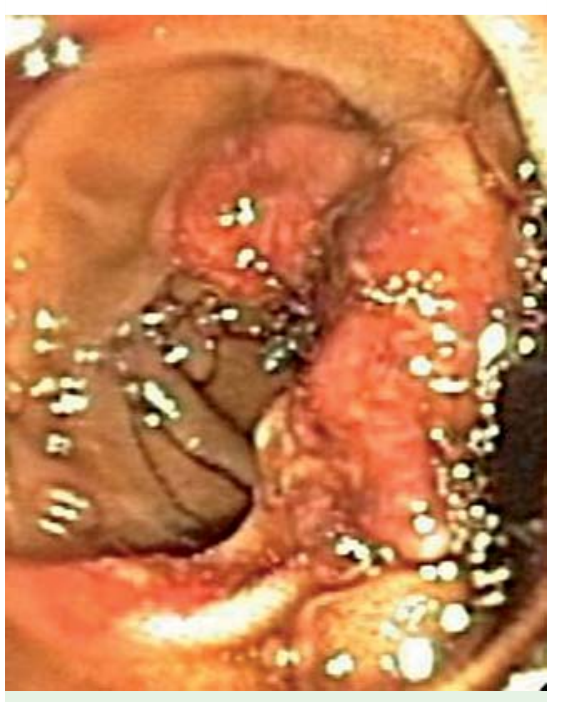

Fig. 1 Endoscopic view of jejunum showing semi-circumferential exophytic bleeding mass.

Primary gastrointestinal angiosarcoma is an extremely rare disease. We present a unique case in which endoscopic examination of the deep small bowel was necessary to make the diagnosis using spiral enteroscopy.

An 82-year-old man with a history of radiation therapy for prostate cancer was referred for persistent, obscure-overt gastrointestinal bleeding. Recent upper endoscopy with push enteroscopy approximately $20 \mathrm{~cm}$ beyond the ligament of Treitz (LOT) was negative. Ileocolonoscopy only revealed blood in the terminal ileum. Computed tomography (CT) angiogram and bleeding scan were negative. The patient received a total of 29 units of packed red blood cells, 10 units of fresh frozen plasma, and 7 units of platelets in the month prior to presentation. On admission to our hospital, his hemoglobin level was $8 \mathrm{~g} / \mathrm{dL}$ with a normal platelet count and prothrombin time. Anterograde (per os) spiral overtube-assisted enteroscopy was performed. The enteroscope was inserted approximately $230 \mathrm{~cm}$ beyond the LOT. Beginning at $40 \mathrm{~cm}$ past the LOT, there were innumerable exophytic, polypoid masses that were oozing blood ranging in size from $5 \mathrm{~mm}$ to $30 \mathrm{~mm}$ (๑ Fig. 1).

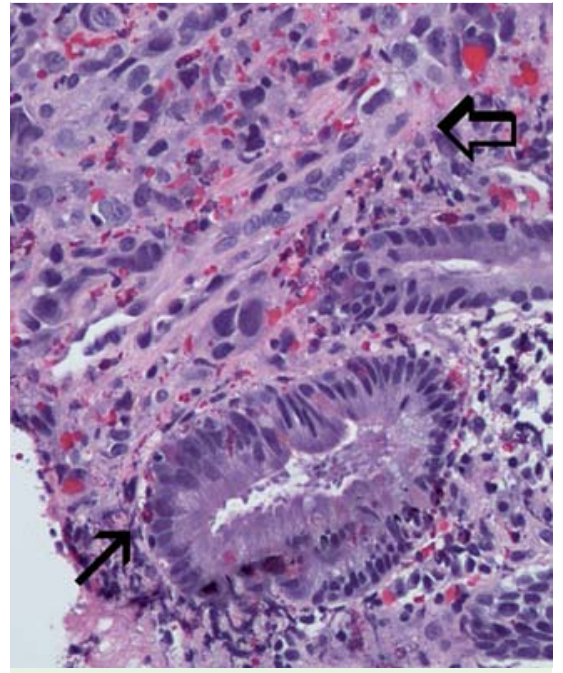

Fig. 2 Low power magnification $(\times 100)$ of small-intestinal lesion showing a normal smallintestinal gland (solid black arrow) and an abnormal vasoformative channel lined with neoplastic epithelioid cells (block arrow).

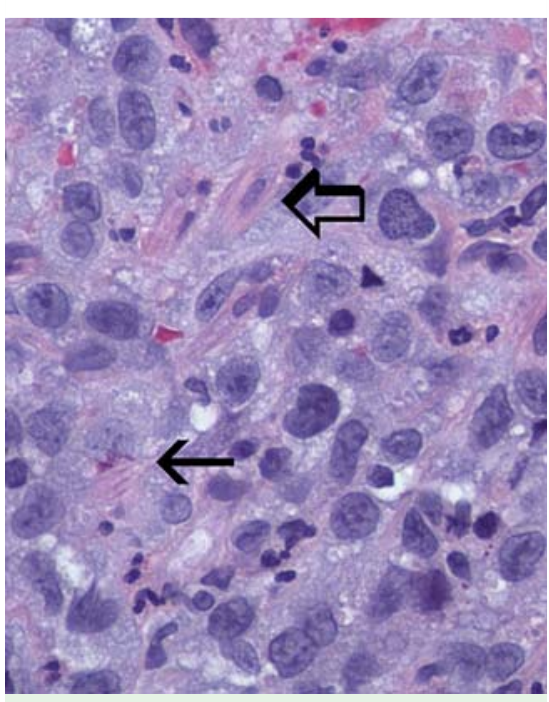

Fig. 3 High power magnification $(\times 400)$ showing an abnormal vasoformative area (solid black arrow) and a plump epithelioid cell with abundant eosinophilic cytoplasm (block arrow).

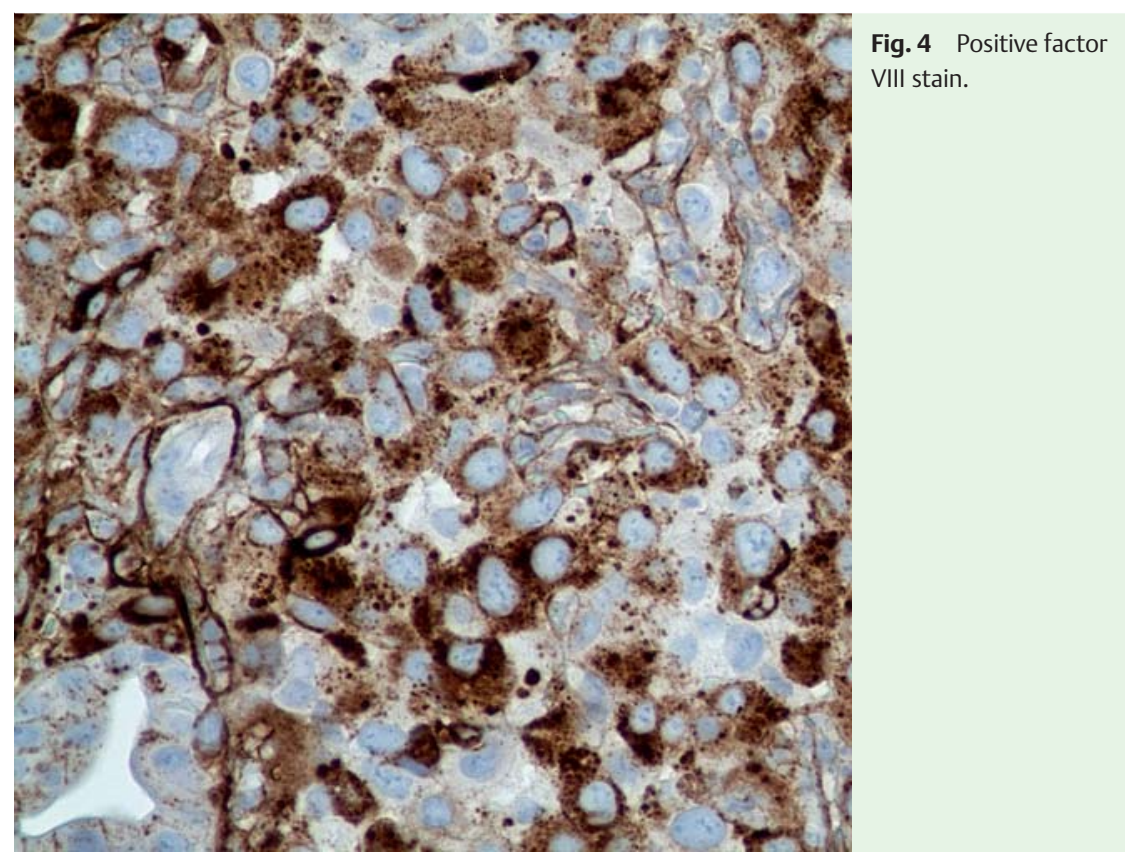

The lesions blanketed the entire jejunum. Multiple biopsies were obtained (๑ Video 1).

Histopathological examination revealed sheets of epithelioid cells with ample eosinophilic cytoplasm containing pleomorphic nuclei ( $\bullet$ Fig. 2, 3).
Frequent mitoses and primitive vascular channels were identified. The diagnosis of angiosarcoma was confirmed by special stains for vimentin, CD31/34 and factor VIII-related antigen ( $\bullet$ Fig. 4). The patient died 6 weeks later from intractable bleeding and exsanguination. 
Angiosarcoma is a rare, highly aggressive vascular neoplasm primarily involving the skin and soft tissues [1]. Infrequently, angiosarcoma may involve the abdominal organs such as the liver, spleen and adrenals. Primary gastrointestinal angiosarcoma, however, is an extremely rare tumor with only 30 cases described in the literature since 1970 [2]. The exact pathogenesis of angiosarcoma remains unclear, but some predisposing factors include previous radiation therapy, exposure to vinyl chloride, Thorotrast or arsenic, and hemodialysis or chronic lymphedema [3-5]. In most cases of gastrointestinal angiosarco$\mathrm{ma}$, the symptoms are nonspecific and include abdominal pain, weight loss, and anorexia. Overt bleeding with melena or hematochezia is less common, and thus tagged red blood cell scintigraphy (bleeding scan) and often angiogram are not useful. Direct visualization and biopsy of suspected intestinal lesions with either push enteroscopy or overtube-assisted deep enteroscopy is the most reliable method of diagnosis. Histopathological examination of gastrointestinal angiosarcoma usually reveals solid areas of epithe-

\section{Video 1}

Endoscopic video of anterograde spiral overtube-assisted enteroscopy showing innumerable lesions in the jejunum, ranging from tiny polypoid lesions to large exophytic masses, which were causing massive overt gastrointestinal bleeding. Biopsies from these lesions confirmed the diagnosis of primary small-bowel angiosarcoma, an extremely rare tumor of the gastrointestinal tract. lioid cells with abundant eosinophilic cytoplasm. In addition, there are vasoformative areas with anastomosing vessels lined by spindle-shaped cells. Immunohistochemical staining with vimentin, CD31/34 and factor VIII-related antigen confirms the vascular nature of this lesion. Stains for cytokeratin (adenocarcinoma), S100 (neuroma or melanoma), c-kit (gastrointestinal stromal tumor) are negative.

Death from gastrointestinal angiosarcoma is often due to exsanguination. Surgical resection offers the best chance for cure if the disease is limited. Currently there are no well-defined chemotherapy regimens for primary gastrointestinal angiosarcoma, as few randomized clinical trials have been performed. Taxol and thalidomide can be considered because of their antiangiogenic properties [6]. Ifosfamide, docetaxel, and recombinant human interleukin-2 have been reported to be successful $[7,8]$.

\section{Endoscopy_UCTN_Code_CCL_1AC_2AC}

\section{Competing interests: None}

V. Jayaraman ${ }^{1}$, M. N. Wilkinson', S. Nagula ${ }^{1}$, M. Siebel ${ }^{2}$, J. C. Bucobo ${ }^{1}$, S. Zee ${ }^{3}$, J. M. Buscaglia ${ }^{1}$

1 Divisions of Gastroenterology, Stony Brook University Medical Center, State University of New York at Stony Brook, Stony Brook, New York, USA

2 Divisions of Oncology, Stony Brook University Medical Center, State University of New York at Stony Brook, Stony Brook, New York, USA

3 Department of Pathology, Stony Brook University Medical Center, State University of New York at Stony Brook, Stony Brook, New York, USA

\section{References}

1 Maddox JC, Evans HL. Angiosarcoma of skin and soft tissue: A study of forty-four cases. Cancer 1985; 48 (8): 1907-1921

2 Grewal JS, Daniel ARM, Carson EJ et al. Rapidly progressive metastatic multicentric epithelioid angiosarcoma of the small bowel. Int J Colorectal Dis 2008; 23 (8): 745 - 756

3 Telles NC, Thomas LB, Popper H et al. Evolution of Thorotrast-induced hepatic angiosarcomas. Environ Res 1979; 18 (1): 74- 87

4 Goette DK, Detlefs RL. Post-irradiation angiosarcoma. J Am Acad Dermatol 1985; 12: 922-926

5 Byers RJ, McMahon RF, Freemont AJ et al. Angiosarcoma at the site of a ligated arteriovenous fistula in a renal transplant recipient. Nephrol Dial Transplant 1994; 9 (1): 112

6 Fraiman G, Ganti AK, Potti A et al. Angiosarcoma of the small intestine: a possible role for thalidomide. Med Oncol 2003; 20 (4): 397-402

7 Nagano T, Yamada Y, Ikeda T et al. Docetaxel: a therapeutic option in the treatment of cutaneous angiosarcoma: report of 9 patients. Cancer 2007; 110: 648-651

8 Inaba T, Yamanaka K, Asahi Ket al. Complete remission in a patient with angiosarcoma by the combination of OK-432, rhIL-2, and radiotherapy. Eur J Dermatol 2005; 15 (5): 411-413

Bibliography

DOI $10.1055 / \mathrm{s}-0030-1256397$

Endoscopy 2011; 43: E219-E220

(c) Georg Thieme Verlag KG Stuttgart · New York . ISSN 0013-726X

Corresponding author

V. Jayaraman

Division of Gastroenterology Stony Brook University Medical Center 100 Nicolls Road

HSC Level 17

Room 060

Stony Brook

NY 11794

Fax: +631-444-8886

vijay.jayaraman@stonybrook.edu 